\title{
Dimension in a Radiative Stellar Atmosphere*
}

\author{
J.P. Krisch and E.N. Glass ${ }^{\dagger}$ \\ Department of Physics, University of Michigan, Ann Arbor, Michgan 48109
}

(31 January 2001, preprint MCTP-01-15)

\begin{abstract}
Dimensional scales are examined in an extended $3+1$ Vaidya atmosphere surrounding a Schwarzschild source. At one scale, the Vaidya null fluid vanishes and the spacetime contains only a single spherical 2-surface. Both of these behaviors can be addressed by including higher dimensions in the spacetime metric.
\end{abstract}

KEY WORDS: Spacetime dimensions; fractals; Kaluza-Klein

\footnotetext{
*The original version of this paper received Honorable Mention as a 2000 Gravity Research Foundation Essay

†Permanent address: Physics Department, University of Windsor, Ontario N9B 3P4, Canada
} 


\section{INTRODUCTION}

The nature and number of dimensions of the Universe are evolving ideas. From the $3+1$ description of Minkowski spacetime, we have come to study theories involving much higher dimensions [4], [2], [3], 困, [5]. There are descriptions of processes requiring non-integer dimensions [6] and negative dimensions can give interesting results in some calculations [7]. Spacetime models in lower dimensions have proved useful in gaining insights to some of the problems of quantum gravity [8]. Identifying observable dimensional effects is one of the critical components to understanding our universe.

The Vaidya spacetime provides a useful laboratory for studying possible effects of fractal dimensions. A generalized Vaidya spacetime with horizon function $m_{(d)}(u)$ and dimension parameter $d$ has metric [9]

$$
d s_{3+1}^{2}=g_{a b}^{V a d}(d) d x^{a} d x^{b}=\left[1-\frac{2 m_{(d)}}{r}\right] d u^{2}+2 d u d r-\left(r / r_{0}\right)^{d-1} r_{0}^{2} d \Omega^{2},
$$

where $r_{0}$ has units of length so that spheres have area of (length) ${ }^{2}$. As we examine radial lines at fractal scales, each $r$ value provides a set of points enclosed by a 2 -sphere with luminosity area proportional to $r^{d-1}$. The metric describes a Schwarzschild object surrounded by a two fluid atmosphere; a radiation fluid and a fluid with density $\Gamma$, radial heat flow $q_{r}$, and radial

and transverse stresses [9], [10]. The atmosphere supports a variety of processes occurring at microscopic scales, and for each of these processes, the metric can be written with an appropriate dimension, $d$. For example, with $d=3$ and $m_{(3)}(u)=m_{0}$, an observer sees the Schwarzschild vacuum. The same observer, looking at smaller scales, i.e. at different $d$ in the same physical spacetime, would see processes characterized by a fractal dimension, different $m_{(d)}$ and non-zero fluid content. If we are observing in a $3+1$ spacetime, the horizon function $m_{(d)}(u)$ measures the system mass only for spatial dimension $d=3$. For 
all $d$, the sectional curvature mass $M$ [10, [11] and density $\Gamma$ describe the content within 2-surfaces of constant $u$ and $r$ :

$$
\begin{aligned}
-\frac{2 M}{r^{3}} & =R_{a b c d} \hat{\vartheta}^{a} \hat{\varphi}^{b} \hat{\vartheta}^{c} \hat{\varphi}^{d}=\frac{(d-1)^{2}}{4 r^{2}}\left[1-\frac{2 m_{(d)}}{r}\right]-\frac{r_{0}^{d-3}}{r^{d-1}}, \\
4 \pi\left(\Gamma-\Gamma_{0}\right) & =r_{0}^{d-3} r^{1-d} \partial_{r} M
\end{aligned}
$$

where $\hat{\vartheta}^{a}=r_{0}^{(d-3) / 2} r^{(1-d) / 2} \delta_{\vartheta}^{a}$ and $\hat{\varphi}^{a}=r_{0}^{(d-3) / 2} r^{(1-d) / 2} \sin ^{-1} \vartheta \delta_{\varphi}^{a}$.

The Raychaudhuri equation, used with null outgoing or incoming generators, determines the focusing of null rays. The existence of an horizon is expected to persist at all scales, since the focusing properties of incoming and outgoing null geodesics transform covariantly with the size of their orthogonal 2-spheres.

The interpretation of the metric parameter $d$ as a fractal dimension follows from considering diffusive processes in the atmosphere. If $\mathcal{P}(u, r)$ is a quantity that is diffusing, the diffusion equation is

$$
\partial_{u} \mathcal{P}=\frac{1}{r^{d-1}} \partial_{r}\left(D r^{d-1} \partial_{r} \mathcal{P}\right)
$$

and $d$ is identified as the dimension of the fractal substrate on which the diffusion is oc-

curring [12], [13]. At smaller quantum levels, $\mathcal{P}$ is a probability measure on the space of radial paths [14. Fractal dimensions are defined and discussed by Hughes [15. Diffusion is a probe of the smaller fractal dimensions because a continuous description of a diffusive process can envelope underlying dimensional behavior [12]. Diffusion observations can provide information about these smaller scales which are not directly accessible to observation.

\section{DIMENSION AND FLUID CONTENT}

The field equations provide a 2-fluid description of the matter and radiation content [10]

$$
\begin{gathered}
8 \pi \rho_{\text {null }}=-\frac{(d-1) \partial_{u} m_{(d)}}{r^{2}}+\frac{(d-1)(3-d)}{8 r^{2}}\left[1-\frac{2 m_{(d)}}{r}\right], \\
8 \pi \rho=\frac{r_{0}^{d-3}}{r^{d-1}}+\frac{(d-1)}{8 r^{2}}\left[11-5 d+\frac{5(d-3) m_{(d)}}{r}\right]
\end{gathered}
$$




$$
\begin{aligned}
8 \pi\left(\rho+p_{r}\right) & =16 \pi q_{r}=\frac{(3-d)(d-1)}{4 r^{2}}\left[1-\frac{2 m_{(d)}}{r}\right], \\
8 \pi p_{\perp} & =-\frac{(d-3)}{r^{2}}\left[\frac{(d-3) m_{(d)}}{2 r}+\frac{d-1}{4 r^{2}}\right] .
\end{aligned}
$$

$\rho$ can be related to $\Gamma$ through $M$. For dimension $d=3$, we have the equivalences $M=m_{(3)}$ and $\Gamma=\rho$.

There are some interesting aspects to the $d=1$ scale. The sectional curvature mass and density are $M=M_{0} r^{3}$, and $\Gamma=\Gamma_{0} r^{2}$. The fluid content is

$$
\begin{aligned}
\rho_{\text {null }} & =q_{r}=0 \\
\rho & =-p_{r}=\frac{1}{8 \pi r_{0}^{2}}, \\
8 \pi p_{\perp} & =-\frac{2 m_{(1)}}{r^{3}} .
\end{aligned}
$$

The null radiation and heat flow vanish while the density and radial stress assume constant values. From the metric

$$
d s_{3+1}^{2}=\left[1-\frac{2 m_{(1)}}{r}\right] d u^{2}+2 d u d r-r_{0}^{2} d \Omega^{2}
$$

one sees that all spheres have the same constant surface area $4 \pi r_{0}^{2}$. In the range of spacetime scales, $d=1$ marks the boundary between a geometry where surface area and circumference increase with radius and one where they decrease with radius.

\section{INVARIANTS}

As an aid to identifying the coordinate independent behavior of fractal dimension $d$, we compute tensor invariants of metric (11). The Ricci scalar is given by

$$
R_{a b} g^{a b}=\frac{2}{r^{2}}\left(\frac{r_{0}}{r}\right)^{d-3}+\frac{m_{(d)}(d-3)(3 d-5)}{r^{3}}+\frac{(1-d)(3 d-7)}{2 r^{2}} .
$$

The Ricci, Weyl, and Riemann quadratic invariants are

$$
\begin{aligned}
R_{a b} R^{a b}= & \frac{2}{r^{4}}+\left(\frac{2}{r_{0}^{4}}\right)\left(\frac{r_{0}}{r}\right)^{2 d-2}+2\left(\frac{d-1}{r_{0}^{5}}\right)\left(\frac{r_{0}}{r}\right)^{d+2}\left[2 m_{(d)}(d-3)-r(d-2)\right] \\
& +\left(\frac{d-3}{4 r^{6}}\right)\left[\begin{array}{c}
4 m_{(d)}^{2}(d-3)\left(3 d^{2}-8 d+7\right)+4 \partial_{u} m_{(d)} r^{2}(d-1)^{2} \\
+r^{2} d\left(3 d^{2}-11 d+15\right)-d m_{(d)} r\left(12 d^{2}-56 d+84\right)+r\left(40 m_{(d)}-3 r\right)
\end{array}\right] .
\end{aligned}
$$




$$
\begin{aligned}
& C_{a b c d} C^{a b c d}=\frac{1}{3 r^{4}}\left[d-1-4 d m_{(d)} / r-2\left(r_{0} / r\right)^{d-3}\right]^{2} . \\
R_{a b c d} R^{a b c d}= & \left(\frac{2}{r_{0}^{2}}\right)^{2}\left(\frac{r_{0}}{r}\right)^{2 d-2}+\left(\frac{m_{(d)}}{r^{3}}\right)^{2}\left[(d-3)\left(3 d^{3}-15 d^{2}+29 d-1\right)+48\right] \\
& +\left(\frac{d-1}{2 r^{2}}\right)^{2}\left(3 d^{2}-14 d+19\right)+\left(\frac{2 \partial_{u} m_{(d)}}{r^{4}}\right)(d-3)(d-1)^{2} \\
& -\left(\frac{m_{(d)}}{r^{5}}\right)(d-1)^{2}\left(3 d^{2}-16 d+25\right)+\left[\frac{2(d-1)^{2}}{r^{5}}\right]\left(\frac{r_{0}}{r}\right)^{d-3}\left(2 m_{(d)}-r\right) .
\end{aligned}
$$

\begin{tabular}{|c|c|c|}
\hline & $\underline{d=1}$ & $\underline{d=3}$ \\
\hline$\underline{R_{a b} g^{a b}}$ & $2 / r_{0}^{2}+4 m_{(1)} / r^{3}$ & 0 \\
\hline$\underline{R_{a b} R^{a b}}$ & $2 / r_{0}^{4}+8 m_{(1)}^{2} / r^{6}$ & 0 \\
\hline$\underline{C_{a b c d} C^{a b c d}}$ & $(1 / 3)\left[2 / r_{0}^{2}+4 m_{(1)} / r^{3}\right]^{2}$ & $48 m_{(3)}^{2} / r^{6}$ \\
\hline$\underline{R_{a b c d} R^{a b c d}}$ & $4 / r_{0}^{4}+16 m_{(1)}^{2} / r^{6}$ & $48 m_{(3)}^{2} / r^{6}$ \\
\hline$\underline{S_{a b} S^{a b}}$ & {$\left[1 / r_{0}^{2}-2 m_{(1)} / r^{3}\right]^{2}$} & 0 \\
\hline$\underline{S_{a b} S_{c}^{b} S^{a c}}$ & 0 & 0 \\
\hline
\end{tabular}

Values of the invariant scalars are tabulated for the $d=3$ Vaidya null fluid and the $d=1$ single sphere space $\left(S_{a b}=R_{a b}-\frac{1}{4} R g_{a b}\right.$ is the trace-free Ricci tensor):

A set of 16 real valued scalar invariants is given by Carminati and McLenaghan [16]. Using GRTensor [17] we have computed the entire set for $d=1$ and $d=3$. The $d=3$ Vaidya spacetime has only 2 non-zero scalars out of 16 , and they are powers of $m_{(3)} / r^{3}$, so $d=3$ has one independent invariant. The $d=1$ space has 9 non-zero Carminati-McLenaghan scalars, all powers of $\left(r^{3} \pm 2 r_{0}^{2} m_{(1)}\right) /\left(r_{0}^{2} r^{3}\right)$. Hence $d=1$ has two independent invariants. None of the scalar invariants place the $d=1$ family in a special category. However, when the optical scalars for the null generators of 2 -spheres are examined then $d=1$ stands out.

\section{OPTICAL SCALARS}

We write metric (迎) as $g_{a b}^{V a d}(d)=2 l_{(a} n_{b)}-2 m_{(a} \bar{m}_{b)}$ using a Newman-Penrose null tetrad where 


$$
\begin{aligned}
l_{a} d x^{a} & =d u \\
n_{a} d x^{a} & =d r+(1 / 2)\left[1-\frac{2 m_{(d)}}{r}\right] d u, \\
m_{a} d x^{a} & =-\left(r_{0} / \sqrt{2}\right)\left(r / r_{0}\right)^{(d-1) / 2}(d \vartheta+i \sin \vartheta d \varphi),
\end{aligned}
$$

with non-zero spin coefficients

$$
\begin{aligned}
& \rho=(1-d) /(2 r), \quad \mu=[(1-d) /(4 r)]\left[1-\frac{2 m_{(d)}}{r}\right] \\
& \alpha=-\left(r_{0} / r\right)^{(d-1) / 2} \cot \vartheta /\left(2 \sqrt{2} r_{0}\right)=-\beta \\
& \gamma=m_{(d)} /\left(2 r^{2}\right) .
\end{aligned}
$$

The only non-zero Weyl tensor component is

$$
\Psi_{2}=-\left(\frac{1}{6 r_{0}^{2}}\right)\left(\frac{r_{0}}{r}\right)^{d-1}+\frac{(d-1) r-4 m_{(d)} d}{12 r^{3}}
$$

identifying the $g_{a b}^{V a d}(d)$ spacetime as Petrov type D. Both $l^{a}$ and $n^{a}$ are tangent to hypersurface orthogonal null geodesics. Spin coefficients $\rho$ and $\mu$ are the optical scalars describing the expansion of the incoming and outgoing null geodesic congruences from two-surfaces of constant $u$ and $r$. When $d=1$, we see from Eq.(13a) that the expansion is zero, and so the $(\vartheta, \varphi)$ two-surface at $r_{0}$ is marginally trapped.

\section{POSSIBILITIES}

There are several possible explanations for the $d=1$ behavior. The first is that $d$ must be greater than one. Eliminating $d \leq 1$ would be necessary if the field equation description were not correct for this range of $d$. The model describes $d$ as a fractal dimension which changes as one goes down in spatial scales. At some scale one might expect fractal functions to provide valid descriptions of physical parameters like a matter distributions. Since fractal functions like the Weierstrass function [18] are continuous, but not differentiable, conventional general relativity would not work [19], [20]. Another possibility is that $d=1$ is only observable in the final state. Lindquist, Schwartz, and Misner [21] have pointed out that Vaidya's $u$ in metric (1) covers the lower half-plane of Kruskal's $(v, w)$ coordinates in Fig.(1) of [21]. 
The Schwarzschild $(T, r)$ sector is the lower quadrant $(v \leq 0, w \geq 0)$. To quote from [21]: "The hypersurface $r=2 m_{(3)}(u \rightarrow \infty)$ in Vaidya's metric is analogous to the Schwarzschild hypersurface $r=2 m_{(3)}(T \rightarrow \infty)$ in Kruskal's metric". It is future null infinity that is the horizon at $r_{0}$. The intersections of outgoing null surfaces with future null infinity are 2 -spheres. Since there is only one 2 -sphere at the $d=1$ scale, this implies that $u \rightarrow \infty$ and the $d=1$ scale is only observable in the late time Vaidya solution when the time dependence of $m_{(3)}(u)$ has vanished. This is the second of the two possibilities mentioned by Lindquist et al. We expect $m_{(3)}(u)$ will evolve toward $m_{(1)}(u \rightarrow \infty)$. The existence of only a single spherical area suggests a new possibility.

From the field equations we see that the density $\rho$ is constant for $d=1$, and $\Gamma \sim r^{2}$. The behavior of these two densities can be reconciled if $r=r_{0}$ and both $M$ and $\Gamma$ are constant. The existence of only one spherical surface suggests that at the $d=1$ scale we have lost a dimension and are looking only at the horizon. One of Mandelbrot's examples [6] of the variability of dimension describes how a ball of thin thread is seen as an observer changes scale. From far away it seems a point, which becomes a 3-dimensional ball at a closer distance. As an observer moves down through various scales, the ball changes to a set of 1-dimensional fibers which become 3-dimensional cylinders, etc. While the embedding dimension for the ball has not changed, the effective dimension of the contents does change. At $d=1$, the effective spatial dimension of the Vaidya spacetime appears to be two. $d=1$ could mark a real drop in dimension with the onset of new physics, or the apparent loss of dimension may imply the existence of dimensions higher than four in the "real" spacetime. The new dimensions would be analogous to the embedding dimensions in Mandelbrot's example. It is possible that there are compact [22] or non-compact [23] dimensions and one begins to see more than four dimensions at $d=1$. At this scale, the $3+1$ metric is simply not correct although general relativity can still provide a valid description. Given the recent interest in higher dimensions in our universe this last explanation is promising, requiring new dimensions but maintaining the formalism of general relativity as an investigative tool. 


\section{HIGHER DIMENSIONS}

Two features which emerge from the $3+1$ analysis are the vanishing of the null fluid at the $d=1$ scale and the existence of a single 2 -sphere for the entire spacetime. Using conventional general relativity, both of these features can be addressed with a higher dimensional metric. Labelling the extra spatial dimension by coordinate $y$, one could have for example,

$$
\begin{aligned}
d s_{4+1}^{2}= & {\left[1-\frac{2 m_{(d)}(u)}{r}\right] d u^{2}+2 d u d r-\left(r / r_{0}\right)^{d-1} r_{0}^{2}\left[d \vartheta^{2}+\sin ^{2} \vartheta d \varphi^{2}\right] } \\
& -\left(r / r_{1}\right)^{2} d y^{2}
\end{aligned}
$$

with $r_{1}$ a dimensional constant similar to $r_{0}$. The extra spatial dimension allows higher dimensional spherical surfaces. Calculating the field equations for $d=1$, the extra dimension provides a non-zero null fluid density containing the term $-\partial_{u} m_{(1)} / r^{2}$. More complicated metrics with $y$ dependant warp factors provide similar results.

The behavior of this system at $d=1$ could be evidence for higher dimensions which begin to be important at the $d=1$ scale. Currently, some theories involving small extra dimension have been studied; for example, the Kaluza-Klein theories with a rolled up 5th dimension, superstring theories with several methods of compactification [24], the gravitational bulk propagation model of Arkani-Hamed et al [25] or the Cantorian spacetime model described by El Naschie [26]. There have also been various ways suggested to search for these extra dimensions.

If the scale of the extra dimensions is of order $1 \mathrm{~mm}$, then there should be evidence in torsion-balance tests [27]. Smaller scales might be found in accelerator experiments. Scat-

tering experiments which could find evidence for extra dimensions have been discussed by groups at SLAC [28] and CERN [29]. On the astrophysical level, Liu et al [30] have discussed some solar system tests based on a 5-dimensional extension of the Schwarzschild metric and Cassisi et al [31] have examined the effects of higher dimensions on stellar evolution. A lower bound on extra dimensions based on light-cone fluctuations has been discussed by Yu and Ford [22]. Evidence for higher dimensions could also show up in a transport process like 
diffusion.

Consider, for example, a diffusive process: let $p(u, r)$ be the probability at time $u$ for an element of the atmosphere to be in a shell between $r$ and $r+d r . p(u, r)$ obeys an anomalous diffusion equation [12]

$$
\partial_{u} p=\frac{1}{\sqrt{-g}} \partial_{r}\left[D \sqrt{-g} \partial_{r} p\right]
$$

with $\sqrt{-g} \sim r^{d}$ and diffusivity $D(r)=D_{0} r^{-\theta}$. The solution is

$$
p(u, r) \sim u^{-(d+1) /(2+\theta)} e^{-c r^{2+\theta} / u} .
$$

From this probability one can show that $\left\langle r^{2}(u)>\sim u^{2 /(2+\theta)}[12]\right.$. The scaling with time samples the connectivity of the substrate through the radial dependence of the diffusivity. There is evidence, in condensed matter calculations, that the exponent $\theta$ depends on the number of spatial dimensions [32]. If this trend carries over to gravitational problems, we would expect to see decreasing time dependence in the scaling relation as the number of dimensions increases. Observations of Vaidya atmospheric scaling behavior could provide information about dimension. Focusing on physical quantities such as fluid density, that are proportional to the probability density, would also allow dimensional effects to be studied.

In conclusion, examining the $3+1$ Vaidya spacetime over decreasing scales has highlighted a scale with features that can be explained, within conventional relativity, by including higher dimensions. Dimensional physics has become an active area of investigation with some promise of future experimental insights [1].

[1] Abel, S.A. (1999) Extra dimensions around the corner, Physics World 12, 21.

[2] Duff, M.J. (1999) TASI lectures on Branes, Black holes and Anti-de-Sitter space, Preprint, hep-th/9912164.

[3] Kan, N. and Shiraishi, K. (1998) Fermion Stars with an Extra Dimension, Preprint, grqc/9806074. 
[4] Gunther, U. and Zhuk, A. (1999) Observable effects from extra dimensions, Preprint, grqc/9905109.

[5] Liu, H. and Wesson, P.S. (1998) Mod. Phys. Lett. A13, 2689.

[6] Mandelbrot, B. (1982) The Fractal Geometry of Nature, (W. H. Freeman, San Francisco).

[7] Duplantier, B. (1988) Statistical Mechanics of Membranes and Surfaces, eds. Nelson, D. Piran, T. and Weinberg, S. (World Scientific, Singapore).

[8] Carlip, S. (1995) Class. Quantum Grav. 12, 2853.

[9] Glass, E.N. and Krisch, J.P. (2000) Class. Quantum Grav. 17, 2611.

[10] Glass, E.N. and Krisch, J.P. (1999) Class. Quantum Grav. 16, 1175.

[11] Glass, E.N. and Krisch, J.P. (2000) Gen. Rel. Grav. 32, 735.

[12] O'Shaughnessy, B. and Procaccia, I. (1985) Phys. Rev. Lett. 54, 455. (1985) Phys. Rev. A32, 3073.

[13] Dekeyser, R. Maritan, A. and Stella, A.L. (1994) Diffusion Processes: Experiment, Theory, Simulations: Proceedings of the Vth Max Born Symposium, (Springer-Verlag, Berlin).

[14] Nelson, E. (1985) Quantum Fluctuations, (Princeton University Press, Princeton).

[15] Hughes, B.D. (1995) Random Walks and Random Environments Vol.1 (Oxford University Press, Oxford).

[16] Carminati, J and McLenaghan, R.G. (1991) J. Math. Phys. 32, 3135.

[17] Musgrave, P. D. Pollney, D. and Lake, K. (2000) GRTensorII, Queens University, Kingston, Ontario.

[18] Berry, M.V. and Lewis, Z.V. (1980) Proc. Roy. Soc. Lond. A 370, 459.

[19] Kobelev, L. Ya. (2000) What Dimensions do time and space have: Integer or Fractional, 
Preprint, arXiv.physics/0001035.

[20] El Naschie, M.S. (2000) Chaos, Solitons and Fractals 11, 1137.

[21] Lindquist, R.W. Schwartz, R.A. and Misner, C.W. (1962) Phys. Rev. 137, B1364.

[22] Yu, H. and Ford, L.H. (1999) Lightcone Fluctuations in quantum gravity and extra dimensions, Preprint, gr-qc/9907037.

[23] Randall, L. and Sundrum, R. (1999) Phys. Rev. Lett. 83, 4690.

[24] Green, M.B. Schwarz, J.H. and Witten, E. (1987) Superstring Theory, (Cambridge University Press, Cambridge).

[25] Arkani-Hamed, N. Dimopoulos, S. and Dvali, G. (1998) Phys. Lett. B429, 263.

[26] El Naschie, M.S. (1998) Chaos, Solitons and Fractals 9, 1445.

[27] Long, J.C. Chan, H.W. and Price, J.C. (1999) Nucl. Phys. B539, 23.

[28] Giudice, G. Rattazzi, R. and Wells, J. (1999) Nucl. Phys. B544, 3.

[29] Mirabelli, E. Perelstein, M. and Peskin, M. (1999) Phys. Rev. Lett. 82, 2236.

[30] Liu, H. Overduin, J. and China, P.R. (2000) Solar System Tests of Higher-Dimensional Gravity, Astrophys. J. (to appear).

[31] Cassisi, S. Castellani, V. Degl'Innocenti, S. Fiorentini, G. and Ricci, B. (2000) Phys. Lett. B481, 323.

[32] Gefen, Y. Aharony, A. and Alexander, S. (1983) Phys. Rev. Lett. 50, 77. 